\title{
MedienPädagogik
}

Zeitschrift für Theorie und Praxis der Medienbildung

Themenheft Nr. 28: Tagungsband: Bildung gemeinsam verändern: Diskussionsbeiträge und Impulse aus Forschung und Praxis. Herausgegeben von David Meinhard, Valentin Dander, Andrea Gumpert, Christoph Rensing, Klaus Rummler und Timo van Treeck.

\section{Medienkompetenz multiplizieren? Entwicklung eines Multiplikator/-innenkonzepts im Lehramtsstudium}

Antje Müller und Mathis Prange

\begin{abstract}
Zusammenfassung
Zur Förderung von Medienkompetenz und medienpädagogischer Kompetenz in der universitären Lehramtsausbildung wurde an der Justus-Liebig-Universität Gießen ein Lehrprojekt initiiert, das an der aktuellen technologischen Ausstattung von deutschen Schulen ansetzt. Als Zugang wurde die Technologie der interaktiven Whitebords gewählt, um gegenwärtige Entwicklungen der Digitalisierung von Unterrichtsräumen zu thematisieren und dabei ausgewählte Aspekte von Medienkompetenz zu fördern. In dem Projekt haben verschiedene Einrichtungen der Universität und der Lehrerbildung kooperiert, um eine Multiplikator/-innenschulung für Studierende zu erarbeiten und zu etablieren. Es wurde eine 28-stündige Qualifizierungsreihe entwickelt, die sich aus modularisierten Workshops zur medientechnischen und -didaktischen Auseinandersetzung mit interaktiven Whiteboards, Elementen der Tutor/-innenausbildung sowie der Erarbeitung eines eigenen Unterrichtsentwurfes zusammensetzt. In dem Artikel werden die entwickelten Module vorgestellt und bewertet, inwieweit sie den von Herzig, Aßmann und Klar (2014) formulierten Zielvorstellungen zur Medienkompetenzförderung für das Lehramtsstudium entsprechen. Im Abgleich mit diesen Zielvorstellungen werden Entwicklungspotentiale des Konzepts identifiziert. Darüber hinaus fliessen Ergebnisse der praktischen Durchführung in Optimierungsvorschläge zur Steigerung der Nachhaltigkeit und Qualität der didaktischen Kompetenzen zum Einsatz von interaktiven Whiteboards in der Lehramtsausbildung - und darüber vermittelt im Schulunterricht - ein.
\end{abstract}

\section{Multiplying media literacy?}

In order to improve media literacy and media education in university teacher training, the University of Giessen initiated a project that focuses on the current level of technical equipment at schools in Germany. Working with interactive whiteboards has been chosen as an approach to tackle the current development of digitalization of classrooms and to improve specific aspects of media competence. Various facilities cooperated to develop and establish a 28 hours training of disseminators consisting of multiple modules which cover media technology and didactics, training for tutors and the development of individual teaching concepts. The following article will introduce these modules and evaluate whether they meet the objectives for media competence development among student teachers as proposed by Herzig, Aßmann and Klar (2014). This evaluation provides 
important information for identifying the development potential the concept offers. Additionally, insights gained from the analysis of coursework can be utilised to develop recommendations for optimising the sustainability and the quality of teaching concepts for the use of interactive whiteboards in teacher education and, ultimately, teaching at schools.

\section{Einleitung}

Medienpädagogische Anteile sind in der universitären Lehramtsbildung noch nicht genügend verankert, wie es angesichts unserer zunehmend mediatisierten Kultur notwendig wäre und von verschiedenen Stellen gefordert wird: Beispielsweise von der Initiative «Keine Bildung ohne Medien!» (Botte et al. 2011), im Positionspapier «Medienkompetenz in der Lehramtsausbildung - Bildung für ein Leben und Arbeiten im 21. Jahrhundert» (Konrad-Adenauer-Stiftung e. V. 2015) oder auch in der Bestandsaufnahme von Röll (2013). Einen Vergleich der einzelnen Bundesländer zum kompetenten Umgang mit digitalen Medien in Schulen bieten z. B. Bos et al. (2015). Eine der zentralen zukünftigen Aufgaben in der universitären Lehrerbildung ist es demnach, Lehramtsstudierende auf eine professionelle - das heisst pädagogisch, fachwissenschaftlich und -didaktisch kundige und reflektierte - Entwicklung und Nutzung neuer Medien im Unterricht vorzubereiten (vgl. Imort und Niesyto 2014). Die Notwendigkeit der pädagogischen Professionalisierung in Bezug auf Medienkompetenz wird seit längerem (vgl. Hugger 1998; Bauer 1999) und in den vergangenen Jahren verstärkt diskutiert (vgl. Niesyto 2014; oder auch Schulz-Zander et al. 2012). Denn gerade schulische Lehrkräfte werden aufgefordert sein, Medienkompetenz selbst aufzuweisen, diese in ihrem Unterricht an Schulen einzubringen und selbst die Rolle einzunehmen, Schüler/-innen Medienkompetenz in unterschiedlichen Handlungsund Inhaltsfeldern zu vermitteln (Herzig et al. 2014, 67-69). Medienkompetenz kann dabei als jene Kompetenz verstanden werden, die es Subjekten ermöglicht, «[...] an einer durch luK-Technologien vermittelten Welt aktiv und verantwortlich teilzunehmen» (Rüsse et al. 2006, 15). Aus Sicht pädagogischer Bemühungen bezeichnet «Medienkompetenz [...] das Bildungsziel, dem eine pädagogisch reflektierte Thematisierung und Nutzung der Neuen Medien im Unterricht sich verpflichtet.» (Rüsse et al. 2006, 15). Doch wie können medienpädagogische Anteile stärker in der universitären Lehramtsbildung verankert werden und welche Rahmenbedingungen müssen dafür etabliert sein? 


\section{Problemstellung und erste Näherung}

Um an die aktuellen Entwicklungen in der pädagogischen Praxis anzuknüpfen, orientierten wir uns an den Gegebenheiten, die Studierende etwa im Rahmen ihrer Schulpraktika in den Klassenräumen vorfinden: Im Zuge der voranschreitenden Digitalisierung von Unterrichtsräumen wurden in den verschiedenen hessischen Regionen in grossem Umfang interaktive Whiteboards (IWBs) angeschafft, die teilweise etablierte Medien wie Schultafel und Overheadprojektor gänzlich abgelöst haben. Sie stellen aus Sicht der hessischen Landesregierung ein zentrales Mittel dar, um Lehrkräfte und Schüler/innen den Zugang zu digitalen Unterrichtseinheiten zu ermöglichen. Dies umfasst insbesondere die Verwendung von Animationen und interaktiven Elementen.

Bislang ist eine umfassende Diskussion über den möglichen Platz dieses Mediums im Unterricht, insbesondere unter Berücksichtigung fachdidaktischer Überlegungen, kaum erfolgt. Darüber hinaus fehlen Studierenden meist die nötigen Konzepte, um den eigenen Unterricht mit IWBs unter didaktischen Aspekten angemessen vorzubereiten und durchzuführen. Mit verschiedenen Handbüchern (vgl. z. B. Caspar 2014; Bohrer und Hoppe 2013; Kohls 2011; Müller 2011) oder digitalen Unterrichtseinheiten für das IWB wird von Seiten der Verlage versucht, auf diesen Trend zu reagieren und gezielt Lehrkräften praxisrelevante Materialien anzubieten. Die Studierenden hingegen treffen in ihren ersten Praktika an Schulen auf diese elektronischen Tafeln, ohne über die nötigen technischen, didaktischen und pädagogischen Konzepte zu verfügen. Eine reflektierte und kritische Auseinandersetzung mit dem Medium und den damit verbundenen Möglichkeiten zum Einsatz im Schulunterricht ist unter diesen Umständen nicht möglich.

Das IWB bietet sich daher als Zugang an, um mit Lehramtsstudierenden - mit direktem Bezug zum Studium - aktuelle Entwicklungen in der Digitalisierung von Unterrichtsräumen zu thematisieren und dabei ausgewählte Aspekte von Medienkompetenz zu fördern.

\section{Medienkompetenz und medienpädagogische Kompetenz}

Der Medienkompetenzbegriff wurde in den letzten Jahrzehnten unterschiedlich diskutiert, akzentuiert und die verschiedenen Facetten des Begriffs wissenschaftlich beleuchtet (vgl. Iske 2015; Moser et al. 2011). In den folgenden Ausführungen beziehen wir uns auf das Medienkompetenzmodell der Paderborner Arbeitsgruppe Medienpädagogik (Tulodziecki et al. 2010), das entwicklungs- und handlungstheoretisch fundiert ist. Das übergeordnete Ziel dieses Ansatzes ist die «Befähigung von Schülerinnen und Schülern zu einem sachgerechten, selbstbestimmten, kreativen und sozial verantwortlichem Handeln in einer von Medien geprägten Welt.» (Tulodziecki et al. 2010,68) Die besondere Eignung des Ansatzes für das vorliegende Praxisprojekt 
ergibt sich einerseits aus den expliziten Bezügen zur allgemeinen Bildungsdiskussion und allgemeinen Didaktik (Tulodziecki et al. 2010, 68ff.). Andererseits ist mit dieser umfassenden Zielformulierung die Hoffnung verbunden, anschlussfähig an fachdidaktische Überlegungen zu sein.

Konkret bezogen auf das Lehramtsstudium wurden von Herzig, Aßmann und Klar (2014) zur Medienkompetenzförderung fünf Zielvorstellungen formuliert:

1. Stärkung der (eigenen) Medienkompetenz einschliesslich der technischen Handhabung von Medien und Informationstechnologien.

2. Erwerb von Kenntnissen zur und Sensibilität für die Bedeutung von Medien für Kinder und Jugendliche.

3. Befähigung zur reflektierten Nutzung von Medien und Informationstechnologien für das Lehren und Lernen.

4. Befähigung zur Wahrnehmung von Erziehungs- und Bildungsaufgaben im Bereich von Medien und Informationstechnologien.

5. Befähigung zur Mitwirkung an der Gestaltung medienpädagogischer Konzepte in der Schule. (Herzig et al. 2014, 69f.).

Die genannten Ziele bieten die Grundlage für Kompetenzbereiche, die spezifisch für die Vorbereitung der Studierenden auf den Schuldienst sind. Sie können über die Medienkompetenz hinaus mit Mediendidaktik, Medienerziehung und medienbezogener Schulentwicklung spezifiziert werden (vgl. Herzig et al. 2014, 70).

\section{Unser Lösungsansatz}

Um die medienpädagogische Kompetenz, einschliesslich der Medienkompetenz, bei angehenden Lehrkräften zu stärken, wurde an der Justus-Liebig-Universität Gießen (JLU) ein Projekt initiiert, das als Lehrinnovation im Rahmen des BMBF-Projekts «Einstieg mit Erfolg» aus Mitteln des "Qualitätspakt Lehre» gefördert wurde. ${ }^{1}$ Das Projekt sah die Erarbeitung und Etablierung einer Multiplikator/-innenschulung für Studierende an der JLU vor. Inhaltlich setzte es an der Arbeit mit IWBs in Schulen an. Angesichts einer sehr grossen Zahl an Lehramtsstudierenden an der JLU (ca. 6.000) und des bereits hohen Workloads innerhalb der Lehramtsstudiengänge, war mit der Ausbildung von studentischen Multiplikator/-innen die Hoffnung verbunden, Wissen und Fähigkeiten im Umgang mit IWBs und deren pädagogisch fundierten Einsatz breitenwirksam im Lehramtsstudium zu verankern.

Die Multiplikator/-innen standen, nachdem sie selbst eine Schulungsreihe zum Umgang und Einsatz mit IWBs durchlaufen hatten, für die Schulung der Zielgruppe der Lehramtsstudierenden im Rahmen ihrer Praktikumsvorbereitung zur Verfügung. Als

1 Zur Entwicklung und Umsetzung kooperierten verschiedene Akteure innerhalb und ausserhalb der JLU. Dazu zählten das Zentrum für Lehrerbildung ( $\mathrm{ZL} L)$, das Hochschulrechenzentrum und das Zentrum für fremdsprachliche und berufsfeldorientierte Kompetenzen (ZfbK) jeweils an der JLU sowie das regionale Medienzentrum (Mauszentrum) als Ansprechpartner für Schulen in der Region Gießen-Vogelsberg. 
Multiplikator/-innen wurden Studierende höherer Semester, Praktikumsbeauftragte und Lehrende angesprochen. Diese wurden in einer 28-stündigen Qualifizierungsreihe (Workshop, eigenständige Erarbeitung eines Unterrichtsentwurfes mit den IWBs, kritische Reflektion von Medieneinsatz, Tutorenausbildung) geschult, um künftig auch die mediendidaktische Auseinandersetzung mit IWBs im Klassenraum mit anderen Studierenden und Praktikumsbeauftragten anleiten zu können. Die Weitergabe des Wissens erfolgte anschliessend im Rahmen von Tutorien, die in Einzelsitzungen während der regulären schulpraktischen Begleitveranstaltungen auf Wunsch der betreuenden Praktikumsbeauftragten stattfanden. Mit diesem Massnahmen sollte Medienkompetenz und medienpädagogische Kompetenz im Rahmen des Studiums «multipliziert» werden und auch auf die Praktikumsphase ausstrahlen.

\section{Umsetzung}

Im Folgenden wird insbesondere auf die einzelnen Phasen der Multiplikator/-innenschulung und der Schulung der Zielgruppe näher eingegangen. Ziel war es, etwa 30 Multiplikator/-innen zu schulen. Es konnten zwar ausreichend Studierende, aber keine - wie geplant - Lehrenden oder Praktikumsbeauftragte für die Schulung gewonnen werden. Die vermuteten Ursachen für diese nicht erreichte Zielgruppe wurden in der Weiterentwicklung des Projektes berücksichtigt (siehe Fazit und Ausblick).

\section{Multiplikator/-innenschulung}

Die Multiplikator/-innenschulung wurde in zwei Gruppen an zwei separaten Terminen durch eine Mitarbeiterin des Hochschulrechenzentrums und je einem auswärtigen Dozenten durchgeführt. In einem zusätzlichen gemeinsamen Termin wurden beide Gruppen zusammengeführt und erhielten eine Schulung aus dem Tutorenprogramm der JLU. An einem weiteren gemeinsamen Termin stellten die Multiplikator/-innen selbst erstellte Konzepte zur Arbeit am IWB vor. Zu deren didaktischer Bewertung waren eine Dozentin der JLU und ein auswärtiger Dozent anwesend.

Dabei wurden neben dem IWB, «klassische» Medien wie Flipchart, Beamer und Pinnwand verwendet. Aus den durchgeführten Schulungen wurden folgende Modulbausteine extrahiert, die nun retrospektiv den oben genannten Zielvorstellungen der Medienkompetenzförderungen zugeordnet werden. Mit diesem Vorgehen sollen sowohl die vorhandenen Potentiale als auch die offenen Punkte in dem vorliegenden Schulungskonzept identifiziert werden. 


\section{- Modul 1 - Technische Grundkenntnisse und Basisfunktionen:}

Dieses Modul dient der ersten Annährung an die Technologie. Es soll Vorwissen aktiviert und es sollen Berührungsängste abgebaut werden, indem die Lernenden direkt mit dem IWB interagieren und gemeinsam die Technik erkunden. Diese technische Einführung lässt sich in der oben genannten Zielvorstellung 1 verorten, indem der Fokus auf die Ausbildung der eigenen Medienkompetenz, insbesondere in Bezug auf die der technischen Bedienung des IWBs liegt.

\section{- Modul 2 - Anregungen und Beispiele für den Einsatz von IWBs im Unterricht:}

In diesem Abschnitt der Schulung sollte zur Reflexion des IWB-Einsatzes im Unterricht anhand konkreter Unterrichtsbeispiele angeregt werden. Es wurden Pro- und Contra-Argumente zum Medieneinsatz in Schulen diskutiert und erste kleinere Unterrichtsideen für den eigenen Unterricht entwickelt. Dieses Modul weist aufgrund seiner reflexiven, diskursiven und produktiven Aspekte Inhalte der Zielvorstellungen 3 und 4 auf. Dies betrifft zum einen die reflektierte Nutzung des IWB im Unterricht und zum anderen die Wahrnehmung von Erziehungs- und Bildungsaufgaben, die mit der mediatisierten Lebenswelt der Schüler/-innen über den Einsatz des IWB im Unterricht hinausweisen bzw. Anknüpfungspunkte für eine breitere Thematisierung von Medien und Informationstechnologien bietet. Letzteres konnte in der vorliegenden Schulung allerdings nicht tiefergehend thematisiert werden.

\section{- Modul 3 - Didaktische Überlegungen zum Einsatz von IWBs im Unterricht:}

Dieser Punkt dient der gezielten didaktischen Reflexion des Einsatzes des IWB und seiner Vor- und Nachteile im Unterricht. Damit kann er wie das zuvor genannte Modul 2 in den Zielvorstellungen 3 und 4 verortet werden. Ziel des Moduls ist, Grundregeln für den IWB-Einsatz zu erarbeiten und zu reflektieren, dass der didaktische Wert des IWB erst im Zusammenspiel mit angemessenen Unterrichtsmethoden und -formen entstehen kann.

\section{- Modul 4 - Rolle von digitalen Medien in der Schule - Irrtümer und Chancen:}

An dieser Stelle steht die Auseinandersetzung mit unterschiedlichen schulpädagogischen Positionen zur Rolle von digitalen Medien im Zentrum. Ebenso wie die Diskussion von notwendigen Voraussetzungen für ihren Einsatz. Damit erweitert dieses Modul den Fokus von IWBs auf weitere digitale Medien in Schule und Unterricht. Es lässt sich daher in den Zielvorstellungen 3 und 4 verorten, je nachdem, wo Schwerpunkte gesetzt werden. Der Fokus liegt in diesem Modul auf Unterricht und der kritischen Auseinandersetzung mit dem Einsatz von Medien. 


\begin{abstract}
- Modul 5 - Vertiefung:
Verwendung von IWB-Software, Peripheriegeräten und alternativer Hardware: Hier werden verschiedene Hard- und Software-Lösungen gegenübergestellt sowie deren Einsatzszenarien und notwendigen Voraussetzungen reflektiert. Das Modul entspricht der Zielvorstellung 1, indem es die technischen Fertigkeiten vertieft und daher im Sinne der fundierten Bedienung einen Teilaspekt der Medienkompetenz abdeckt.
\end{abstract}

\title{
- Modul 6 - Entwicklung eigener Unterrichtsmaterialien für den Einsatz des IWBs:
}

Das Modul 6 hat zum Ziel, die Teilnehmenden eigene Unterrichtsentwürfe und -materialien unter dem Grundsatz «Didaktik vor Methodik» erstellen zu lassen. Sie orientieren sich dabei an formulierten Leitfragen und präsentieren in einer gemeinsamen Vorstellungsrunde ihre Ergebnisse und stellen sie zur Diskussion. In diesem Modul werden mediendidaktische Anteile durch die eigene Anwendung vertieft. Damit dient dieses Modul dazu, Zielvorstellungen 3 und 4 zu erreichen, indem Unterricht selbst aktiv mit Medien gestaltet wird. In der Gruppe werden die erstellten Konzepte diskutiert und können so von allen reflektiert werden. Damit erhalten die Studierenden eine grosse Anzahl an Ideen und kritischen Impulsen, die sie auf ihre eigene Lehre transferieren können.

Auf Basis der hier dargestellten Multiplikator/-innenschulung wurde ein detailliertes und modularisiertes Schulungskonzept sowie entsprechende Begleitmaterialien für die Zielgruppe der Lehramtsstudierenden erarbeitet. Auf dieses können sowohl die geschulten Multiplikatoren als auch die beteiligten Akteure in der Hochschule - über die Projektlaufzeit hinaus - zurückgreifen.

\begin{abstract}
Abgleich der Zielvorstellungen
Im Abgleich mit den Zielvorstellungen medienpädagogischer Kompetenzen zeigt sich, dass insbesondere die ersten vier Zielvorstellungen abgedeckt werden konnten, wobei der Schwerpunkt auf der technischen Bedienung und Stärkung der Medienkompetenz (Zielvorstellung 1), der Befähigung zur reflektierten Nutzung der Technologie für das Lehren und Lernen (Zielvorstellung 3) sowie der Wahrnehmung von Erziehungs- und Bildungsaufgaben im Bereich von Medien und Informationstechnologien (Zielvorstellung 4) liegt. Damit besteht Bedarf, den Erwerb von Kenntnissen zur und Sensibilität für die Bedeutung von Medien für Kinder und Jugendliche (Zielvorstellung 2) und Befähigung zur Mitwirkung an der Gestaltung medienpädagogischer Konzepte in der Schule (Zielvorstellung 5) zu stärken und hier gezielt ergänzende Module zu entwickeln. Indem beispielsweise an der Bedeutung von Medien
\end{abstract}


für Kinder und Jugendliche in ihrer Lebenswelt angesetzt wird (Zielvorstellung 2), bieten sie Anknüpfungspunkte für weitere Lernprozesse innerhalb der Schule. Ebenso sollte behandelt werden, wie über den Unterricht hinaus Medienarbeit an einer Schule gestaltet werden kann. Als Ansprechpartner für die Entwicklung von Medienkompetenzkonzepten für Schulen liessen sich an dieser Stelle auch Medienzentren in die Schulungen mit einbeziehen. Im vorliegenden Fall könnte das Mauszentrum als regionale Medienstelle für Gießen und dem Vogelsbergkreis Teile eines entsprechenden Moduls gestalten.

\section{Ergebnis und Grenzen des Projektes}

\section{Schulung der Multiplikator/-innen}

Für die Schulungen von Studierenden im Rahmen von Praktikumsvorbereitungsgruppen, standen schliesslich acht studentische Multiplikator/-innen zur Verfügung. Diese Gruppe erwies sich als hoch motiviert und engagiert. Allerdings liessen sich weder Lehrende, noch Praktikumsbeauftragte für die Rolle als Multiplikator/-innen gewinnen.

Es konnten auch nur wenige Multiplikator/-innen über einen längeren Zeitraum an das Projekt gebunden werden. Aufgrund zeitlicher Belastung durch Studium und Nebenjobs standen viele von ihnen nur für wenige Schulungen zur Verfügung und erklärten nach wenigen Monaten, nicht länger als Multiplikator/-innen arbeiten zu können. Die verbliebenen Multiplikator/-innen engagierten sich jedoch weit über die Projektdauer hinaus sehr aktiv.

\section{Schulung der Zielgruppe}

Insgesamt wurden 20 Tutorien mit jeweils zwischen 10 und 20 Studierenden von unterschiedlicher Dauer von den Multiplikator/-innen durchgeführt. Es wurden unterschiedliche Module geschult, welche je nach Zeitbudget und thematischem Interesse der zu schulenden Gruppe ausgewählt wurden. Aufgrund von Termin- und Raumproblemen konnten nicht alle interessierten Praktikumsvorbereitungsgruppen an den Tutorien teilnehmen. Darin zeigte sich ein dringender Bedarf an einer entsprechenden technischen Ausstattung der zur Verfügung stehenden Seminarräume. 


\section{Fazit und Ausblick}

Unsere Ausgangsfrage lautete: Lässt sich Medienkompetenz «multiplizieren»? Nach den bisherigen Erfahrungen erweist sich das IWB als geeigneter Einstieg, Medienkompetenz und medienpädagogische Kompetenz zu vermitteln. Die entwickelten Module entsprechen zu einem Grossteil den geforderten Zielvorstellungen der Medienkompetenzförderung nach dem Modell von Herzig, Aßmann und Klar (2014). Ergänzungsbedarf besteht in den Bereichen der Kenntnisse ausserschulischer Mediennutzung - einschliesslich der Bedeutung von Medien für Kinder und Jugendliche - sowie die Befähigung der angehenden Lehrkräfte zur Partizipation an medienbezogener Schulentwicklung.

Für die vorhandenen studentischen Multiplikator/-innen wäre es zentral, dass die nötigen Voraussetzungen für die Multiplikator/-innen geschaffen werden, ihr Wissen auch an Studierende weitergeben zu können. So ist eine Schulung ohne ein interaktives Whiteboard oder vergleichbares Gerät nicht möglich. Während der Projektlaufzeit standen lediglich zwei Räume, die mit einem entsprechenden Gerät ausgestattet waren, für eingeschränkte Zeiten zur Verfügung. Somit konnten nicht alle Praktikumsbeauftragten, die an den Schulungen Interesse hatten, mit ihren Studierendengruppen auch an diesen teilnehmen. Als Problem erwies sich auch, dass Studierende, die eine entsprechende Schulung durch die Multiplikator/-innen erhalten hatten, aufgrund mangelnder Ausstattung der Seminarräume kaum Gelegenheit hatten, ihre erworbenen Kompetenzen im Rahmen ihrer regulären Lehrveranstaltungen anzuwenden oder zu vertiefen.

Bezüglich der studentischen Multiplikator/-innen müsste eine grössere Verbindlichkeit der Weitergabe ihres Wissens z. B. im Rahmen von Hilfskraftverträgen, erreicht werden, um deren längerfristiges Engagement zu gewährleisten. Des Weiteren müssten regelmässige Anreize für die Multiplikator/-innen zur Verfügung stehen (Ergänzungsschulungen, Zugang zu Material und Software, Zugang zu Folgeprojekten etc.), ihre Tätigkeit über einen längeren Zeitraum auszuüben.

Als weitere Rahmenbedingung für eine Steigerung der Nachhaltigkeit, wie auch der Qualität der didaktischen Kompetenzen zum Einsatz der IWBs im Schulunterricht, ist eine engere Verzahnung mit den Fachdidaktiken. Sicherlich können studentische Multiplikator/-innen nur die Zielvorstellung 1 selbstständig vermitteln. Alle anderen Kompetenzen können nur in enger Kooperation mit Lehrkräften in Veranstaltungen erlangt werden. Daher sollten erstens zumindest einzelne Räumlichkeiten der Fachdidaktiken entsprechend ausgestattet werden. Zweitens sollten Lehrende selbst geschult und durch die Multiplikator/-innen dabei unterstützt werden, Studierende im Rahmen regulärer Lehrveranstaltungen an den IWBs zu schulen. Studierende könnten dann unter fachdidaktischer Begleitung ihre Kompetenzen im Einsatz des IWBs im Rahmen ihrer fachdidaktischen Lehrveranstaltungen vertiefen. Ein entsprechendes Projekt ist zurzeit an der JLU in der Umsetzungsphase. 


\section{Literatur}

Bauer, Rudolf. 1999. «Medienkompetenz der Lehrer/-innen: ein Baustein zur Professionalisierung des Lehrerberufs.» In Multimediales Lernen, herausgegeben von Franz Lehner und UIrich Nikolaus, 3-24. Wiesbaden: Springer.

Bohrer, Clemens, und Christian Hoppe. 2013. Interaktive Whiteboards in Hochschule und Schule. München: kopaed.

Bos, Wilfried, Ramona Lorenz, Manuela Endberg, Heike Schaumburg, Renate Schulz-Zander und Martin Senkbeil. 2015. Schule digital - der Länderindikator 2015. Vertiefende Analysen zur schulischen Nutzung digitaler Medien im Bundesländervergleich. Münster: Waxmann.

Botte, Alexander, Kerstin Mayrberger, Horst Niesyto, und Dieter Spanhel. 2011. Keine Bildung ohne Medien! Bildungspolitische Forderungen - Medienpädagogischer Kongress 2011. http://www.keine-bildung-ohne-medien.de/kongress-dokumentation/keine-bildung-ohne-medien_bildungspolitische-forderungen.pdf.

Caspar, Pascal. 2014. Whiteboards in der Schule. Der Einsatz interaktiver Tafeln im Unterricht. Marburg: Tectum Verlag.

Herzig, Bardo, Sandra Aßmann, und Tilman-Mathies Klar. 2014. "Grundbildung Medien im Profilstudium im Lehramt.» In Grundbildung Medien in pädagogischen Studiengängen, herausgegeben von Peter Imort und Horst Niesyto, 65-80. München: kopaed.

Hugger, Kai-Uwe (1998). «Medienpädagogische Professionalität und Medienkritik.» In Technologiekritik und Medienpädagogik, herausgegeben von Theo Hug, 155-166. Baltmannsweiler: Schneider.

Kohls, Christian. 2011. Mein SMART Board. Erfurt: Projekt Bildung Media.

Imort, Peter, und Horst Niesyto. Hrsg. 2014. Grundbildung Medien in pädagogischen Studiengängen. München: kopaed.

Iske, Stefan. 2015. «Medienbildung.»In Medienpädagogik - ein Überblick, herausgegeben von Friederike von Gross, Dorothee M. Meister und Uwe Sander, 247-272. Weinheim und Basel: Beltz Juventa.

Moser, Heinz, Petra Grell, und Horst Niesyto. Hrsg. 2011. Medienbildung und Medienkompetenz: Beiträge zu Schlüsselbegriffen der Medienpädagogik. München: Kopaed. doi:10.21240/ mpaed/20.X.

Müller, Sina. 2011. Das interaktive Whiteboard im Klassenzimmer - und jetzt? Informationen und Einsatzmöglichkeiten. Mülheim an der Ruhr: Verlag an der Ruhr.

Niesyto, Horst. 2014. «Medienpädagogische Professionalisierung, Berufsbild und Berufschancen.» In Prekarisierung der Pädagogik - Pädagogische Prekarisierung?, herausgegeben von Fabian Kessl, Andreas Polutta, Isabell van Ackeren Rolf Dobischat und Werner Thole, 114128. Weinheim und Basel: Beltz Juventa.

Konrad-Adenauer-Stiftung e. V. Hrsg. 2015. Positionspapier: Medienkompetenz in der Lehramtsausbildung. http://www.kas.de/wf/doc/kas_40112-544-1-30.pdf. 
Schulz-Zander, Renate, Birgit Eickelmann, Heinz Moser, Horst Niesyto, und Petra Grell. Hrsg. 2012. Jahrbuch Medienpädagogik 9, Wiesbaden: Springer Verlag.

Röll, Franz Josef. 2013. «Fachkräfte als Zielgruppe / Aus- und Weiterbildung von pädagogischen Fachkräften.» In Medienkompetenzförderung von Kindern und Jugendlichen, herausgegeben vom Bundesministerium für Familie, Senioren, Frauen \& Jugend, 84-94. Berlin: Publikationsversand der Bundesregierung.

Rüsse, Wilfried, Werner Sesink, und Thomas Trebing. 2006. ICuM-Abschlussbericht: Pilotprojekt «ICUM»: IT-Curriculum zur Förderung der Medienkompetenz in Lehramtsstudiengängen.

Tulodziecki, Gerhard, Bardo Herzig, und Silke Grafe. 2010. Medienbildung in Schule und Unterricht: Grundlagen und Beispiele. UTB 3414. Bad Heilbrunn: Julius Klinkhardt. 